Ninth Conference on the Application of Accelerators in Research and Industry

ISOTOPE MATERIALS AVAILABILITY AND SERVICES FOR TARGET PRODUCTION AT THE OAK RIDGE NATIONAL LABORATORY* J. E. Ratledge, Oak Ridge National Laboratory. 1 (30 min.)

Materials available through the Isotope Distribution Program include separated stable isotopes, byproduct radioisotopes, and research quantities of source and special nuclear materials. Isotope products are routinely available in the forms listed in the product description section of the Isotopes Products and Services Catalog distributed by the Oak Ridge National Laboratory (ORNL). Different forms can be provided in some cases, usually at additional cost. Routinely available services include cyclotron target irradiations, fabrication of special physical forms, source encapsulation, ion implantation, and special purifications. Materials and services that are not offered as part of the routine distribution program may be made available from commercial sources in the United States.

Specific forms of isotopic research materials include thin films and foils for use as accelerator targets, metal or other compounds in the form of bars or wires, and metal sheets. Methods of fabrication include evaporation, sputtering, rolling, electrolytic deposition, pressing, sintering, and casting. High-purity metal forms of plutonium, americium, and curium are prepared by vacuum reduction/distillation. Both fissionable and nonfissionable neutron dosimeters are prepared for determining the neutron energy spectra, flux, and fluence at various locations within a reactor.

Details on what materials are available and how the materials and related services can be obtained from ORNL are described.

*Research sponsored by the Division of Environmental Research and Development, U.S. Department of Energy, under contract DE-AC05-840R21400 with the Martin Marietta Energy Systems, Inc.

1T. L. Dah1, C. L. Ottinger, H. S. Aaron, and H. L. Adair, Co-authors, Operations Division, Oak Ridge National Laboratory. 
INTRODUCTION

The Isotope Distribution Program (IDP) at the Oak Ridge National Laboratory (ORNL) is a group of individual activities with separate and diverse DOE sponsors that share the common functions of the production and distribution of isotope products and the performance of isotope-related services. A though no formal charter for the IDP exists, its basic mission is to provide isotope products and associated services to the user community by utilizing government-owned facilities. The basic nolicies under which the IDP is conducted are as follows:

o The program will operate at no cost to the government.

o The materials and services provided through the program will be limited to those that are not produced and reasonably available from domestic private sources.

o The operation will not adversely influence any of DOE's research and development programs.

The IDP is in its 40 th year of operation. Initially, the program provided research quantities of radioactive materials, and through the 1950 's, it was the major supplier of radioisotopes for both research and commercial application. Distribution of enriched stable isotopes began in 1954 . As private production of radioisotopes increased, the government withdrew from production, except for those products which private producers did not supply. 
The IDP currently provides over 300 individual isotope products and associated services to a wide and varied international market. Ultimate applications of the products include health care, research in virtually every scientific discipline, education, industrial use, and national defense. Prices for the products and services are established to recover actual costs, a representative fraction of the government's investment in capital facilities, and an appropriate share of DOE administrative expense. The initial funding is provided through the budgets of various DOE programs, which are credited when the costs of sales transactions are recorcied. The revenues realized from sales are returned as an appropriation reimbursement.

Except for some selected stable isotopes distributed through DOE's Mound Laboratory, production and distribution of the isotope products and services are administered by the Oak Ridge Operations office through the Oak Ridge National Laboratory. Several field offices and DOE sites participate in the program by providing finished products and/or raw materials. 
The IDP consists of the production and distribution of four major categories of isotope products and the perforinance of related services. In all, there are over 300 distinct products and services routinely offered, each with its individual characteristics, uses, markets, etc. 0ther products and/or services are provided under special circumstances. The major categories of isotopes defined for accounting and inanagement purposes are Radioisotopes, Electromagnetically (EM) Enriched Stable I sotopes, Non-EM Stable Isotopes, and Heavy Elements (also called Other Special Materials).

Radioisotopes include specifically produced radioactive materials and byproduct materials with atomic numbers between 1 and 83 . The radioisotopes offered vary widely in half-life, physical/chemical form, and production batch size. The pressent radioisotope product line includes 35 isotopes which represent the remainder of a line which at one time included approximately 15i different isotopes; the others were withdrawn in favor of private producers. However, 20 new isotopes have been added in recent years. Services associated with radioisotopes include encapsulation, packaging, special chemical processing, fabrication of special physical forms, and irradiation services.

Electromagnetic stable isotopes are separated in the Electromagnetic Isotope Enrichment Facility at ORNL, and each isotope is inventoried in a standard chemical form. The quantities produced, enrichinents, and prices vary widely among the 235 different EM stable isotopes sold. In general, buyers prefer the highest enrichment of the respective isotopes. In some cases, however, the requirement is to reduce the concentration of a specific isotope of the element to the lowest possible value. Separation campaigns are usually directed toward the production of one primary isotope of an 
element, with other isotopes of that element also being recovered if needed for the stable isotopes inventory. Services related to EM stable isotopes include conversion to noninventory forms, special purifications, and fabrication of specialized physical forms.

Non-EM stable isotopes are no longer produced by the DOE. Sales of the four isotopes in this category ( $L i-6, L i-7, B-10$, and $B-11)$ are made from existing inventories located at ORNL and at other sites. Services related to non-EM stable isotopes are similar to those for EM materials.

The category of isotopes referred to as Heavy Elements (HE) includes radioactive isotopes with atomic numbers greater than 83. Most of the $22 \mathrm{HE}$ isotopes offered are used for research; however, some have industrial uses. Services include special chemical separations, fabrication of targets and other physical forms, and packaging.

Production of radioisotopes and HE products is conducted at various DOE sites. Radioactive products include byproducts of other operations as well as materials produced specifically for the IDP using reactors and accelerators. In some cases, the finished products are shipped directly to the buyer from the production site, and in other cases, the products are shipped to ORNL for distribution.

Other than the fact that they are referred to as "isotopes," the only common characteristic shared by all products offered under this program is that they are not produced and reasonably available from domestic private sources. The stable isotopes, most of the HE products, and a few of the radioisotopes are amenable to producticn, storage, and distribution on an inventory basis, but many radioactive 
isotopes must be produced frequently or on demand because of rapid decay. There are no prepackaged quantities, so each order must be handled individually. Stable isotopes are usually sold in solid form, but radioactive isotope products may be solid, liquid, or gas. Stable isotopes and most HE products are sold by weight (milligrams or grams), while radioisotopes are sold by the quantity of radioactivity (microcuries, millicuries, or curies). The services performed in the IDP are customerspecific. Each service represents an individual transaction with its own specifications, schedules, etc.

The DOE maintains a separate inventory of highly enriched EM stable isotope products, referred to as the Research Materials Collection (RMC). These products, and some radioactive materials in special circumstances, are loaned for various periods of time to the scientific community for nondestructive experiments. The loanee pays for the work necessary to prepare samples and may al so pay for the use of the material; the latter depending on the government's interest in the specific experiment. The RMc and other loaned materials are not produced under the IDP, but the transactions are handled by the IDP staff. 
ORNL operates the Isotope Research Materials Laboratory (IRML) to provide special research forms of various separated stable isotopes and radioisotopes that are not available from U.S. private suppliers. These special forms include, but are not limited to, compounds in the form of bars or wires, and metal sheets. Methods of fabrication include evaporation, rolling, electrolytic deposition, pressing, sintering, casting, and others. Special purifications can be done by chemical means or by such techniques as crystal-bar preparation (van Arkel-de Boer Process), zone refining and levitation melting. In some cases, single-crystal preparation is possible either in thin-film or bulk form. High-purity metal forms of plutonium, americium, and curium can be prepared by vacuum rediction/ distillation using thorium as the reductant; yields of approximately $75 \%$ for Pu and Am and 50 to $60 \%$ for $\mathrm{Cm}$ are typical.

In addition to research samples, IRML provides neutron dosimetry materials for determining neutron energy spectra, flux, and fluence at various locations within a reactor. Both fissionable and nonfissionable materials are available for monitoring the neutron energy range of interest. Most of the materials are available as ceramic oxide "wires" and are encapsulated in either 1.27-mm or 0.89-mm diam by 3.05-mm- to $8.64-\mathrm{mm}-10$ ng varadium capsules. Each capsule is individually identified, and the encapsulated material is accurately characterized.

As a guide to users, lists of targets and neutron dosimeters made by IRML are shown in Table 1 . Many preparations are not listed, and the ranges noted can be expanded depending on sample size and shape. 
Table 1. Special research material prepared by IRML at ORNL1.

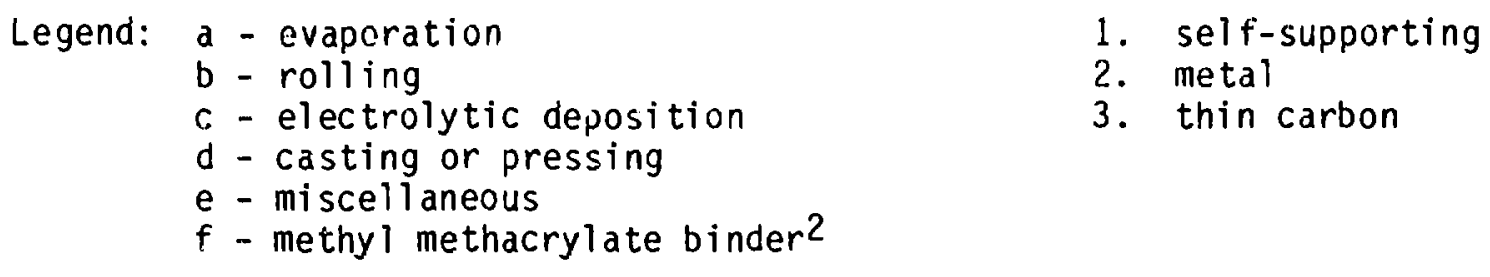

Element

Thickness Range
$\mathrm{g} / \mathrm{cm}^{2}$

Method of

Preparation

Backing

Form of

Deposit

\begin{tabular}{|c|c|c|c|c|}
\hline Aluminum & $\begin{array}{l}5 \text { to } 12,000 \\
>150 \\
\geq 1000\end{array}$ & $\begin{array}{l}a \\
a \\
d\end{array}$ & $\begin{array}{l}2 \\
1 \\
1\end{array}$ & $\begin{array}{l}\text { El ement } \\
\text { El ement } \\
\text { El ement }\end{array}$ \\
\hline Americium & $\frac{>1}{\text { Variable }}$ & $\begin{array}{l}a \\
d\end{array}$ & $\begin{array}{l}2 \\
1\end{array}$ & $\begin{array}{l}\text { Element or oxide } \\
\text { Element or oxide }\end{array}$ \\
\hline Antimony & $\begin{array}{l}>10 \\
500 \text { to } 1000\end{array}$ & $\begin{array}{l}a \\
a\end{array}$ & $\begin{array}{l}2 \\
1\end{array}$ & $\begin{array}{l}\text { El ement } \\
\text { Element }\end{array}$ \\
\hline Arsenic & 10 to 200 & $a$ & 2 & El ement \\
\hline Barium & $\begin{array}{l}10 \text { to } 1000 \\
>2000 \\
\text { Variable }\end{array}$ & $\begin{array}{l}a \\
b \\
d\end{array}$ & $\begin{array}{l}2 \\
1 \\
1\end{array}$ & $\begin{array}{l}\text { Element or salt } \\
\text { clement } \\
\text { Salt }\end{array}$ \\
\hline Beryllium & Variable & $a$ & $1,2,3$ & Element \\
\hline Bi smuth & $\begin{array}{l}>10 \\
500 \text { to } 15,000\end{array}$ & $\begin{array}{l}a \\
a\end{array}$ & $\begin{array}{l}2 \\
1\end{array}$ & $\begin{array}{l}\text { El ement } \\
\text { El lement }\end{array}$ \\
\hline Boron & $\begin{array}{l}10 \text { to } 1000 \\
20 \text { to } 100 \\
1,000 \text { to } 25,000 \\
\text { Variable }\end{array}$ & $\begin{array}{l}a \\
a \\
f \\
d\end{array}$ & $\begin{array}{l}1 \\
2 \\
1 \\
1\end{array}$ & $\begin{array}{l}\text { El ement } \\
\text { El ement } \\
\text { El ement in plastic } \\
\text { El ement }\end{array}$ \\
\hline Bromine & $\begin{array}{l}10 \text { to } 1000 \\
>5000\end{array}$ & $\begin{array}{l}a \\
d\end{array}$ & $\begin{array}{l}2 \\
1\end{array}$ & $\begin{array}{l}\text { Salt } \\
\text { Salt }\end{array}$ \\
\hline
\end{tabular}

${ }^{1}$ Target size, price, and delivery schedule are available upon request. Rolled foils are usually $2 \times 2 \mathrm{~cm}^{2}$.

2hot pressing or rolling. 
Table 1. Continued.

\begin{tabular}{|c|c|c|c|c|}
\hline Element & $\begin{array}{c}\text { Thickness Range } \\
\mathrm{g} / \mathrm{cm}^{2}\end{array}$ & $\begin{array}{l}\text { Method of } \\
\text { Preparation }\end{array}$ & Bacring & $\begin{array}{l}\text { Form of } \\
\text { Deposit }\end{array}$ \\
\hline Cadmium & $\begin{array}{l}10 \text { t.o } 200 \\
>1500 \\
\text { Variable }\end{array}$ & $\begin{array}{l}a \\
b \\
d\end{array}$ & $\begin{array}{l}2 \\
1 \\
1\end{array}$ & $\begin{array}{l}\text { El ement } \\
\text { El ement } \\
\text { El ement }\end{array}$ \\
\hline Calcium & $\begin{array}{l}10 \text { to } 1000 \\
>700 \\
\text { Variable }\end{array}$ & $\begin{array}{l}a \\
b \\
d\end{array}$ & $\begin{array}{l}2 \\
1 \\
1\end{array}$ & $\begin{array}{l}\text { Element } \\
\text { Element } \\
\text { Element or carbonate }\end{array}$ \\
\hline Cal ifornium & $10^{-6}$ to 1.0 & $e$ & 2,3 & oxychloride \\
\hline $\begin{array}{l}\text { Carbon } \\
\quad(\text { normal) }\end{array}$ & $\begin{array}{l}5 \text { to } 200 \\
\geq 50,000\end{array}$ & $\mathrm{a}$ & $\begin{array}{l}1 \\
1\end{array}$ & $\begin{array}{l}\text { El ement } \\
\text { El ement }\end{array}$ \\
\hline Cerium & $\begin{array}{l}>10 \\
>1000 \\
\text { Variable }\end{array}$ & $\begin{array}{l}a \\
b \\
d\end{array}$ & $\begin{array}{l}2 \\
1 \\
1\end{array}$ & $\begin{array}{l}\text { Element or oxide } \\
\text { Element } \\
\text { Element or oxide }\end{array}$ \\
\hline Cesium & 10 to 1000 & $\mathrm{a}$ & 2 & Salt \\
\hline Chlorine & $\begin{array}{l}10 \text { to } 1000 \\
\geq 5000\end{array}$ & $\begin{array}{l}a \\
d\end{array}$ & $\begin{array}{l}2 \\
1\end{array}$ & $\begin{array}{l}\text { Salt } \\
\text { Salt }\end{array}$ \\
\hline Chromium & $\begin{array}{l}>10 \\
>300\end{array}$ & $\stackrel{a, c}{c}$ & $\begin{array}{l}2 \\
1\end{array}$ & $\begin{array}{l}\text { Element } \\
\text { Element }\end{array}$ \\
\hline Cobalt & $\begin{array}{l}>10 \\
100 \text { to } 1000 \\
>1000 \\
\text { Variable }\end{array}$ & $\begin{array}{l}a \\
a \\
b \\
d\end{array}$ & $\begin{array}{l}2 \\
1 \\
1 \\
i\end{array}$ & $\begin{array}{l}\text { El ement } \\
\text { El ement } \\
\text { El ement } \\
\text { El ement }\end{array}$ \\
\hline Copper & $\begin{array}{l}10 \text { to } 1000 \\
>200 \\
>700 \\
\text { Variable }\end{array}$ & $\begin{array}{l}c \\
a \\
b \\
d\end{array}$ & $\begin{array}{l}? \\
1 \\
1\end{array}$ & $\begin{array}{l}\text { El ement } \\
\text { El ement } \\
\text { El ement } \\
\text { El ement }\end{array}$ \\
\hline Curium & $\begin{array}{l}>10^{-3} \\
\text { Variable }\end{array}$ & $\begin{array}{l}a \\
d\end{array}$ & 2,3 & $\begin{array}{l}\text { Oxide } \\
\text { Oxide }\end{array}$ \\
\hline Dysprosium & $\begin{array}{l}>10 \\
>1000 \\
\text { Variable }\end{array}$ & $\begin{array}{l}a \\
b \\
d\end{array}$ & $\begin{array}{l}2 \\
1 \\
1\end{array}$ & $\begin{array}{l}\text { Element } \\
\text { El ement } \\
\text { Element or oxide }\end{array}$ \\
\hline Erbium & $\begin{array}{l}>10 \\
>1000 \\
\text { Variable }\end{array}$ & $\begin{array}{l}a \\
b \\
d\end{array}$ & $\begin{array}{l}2 \\
1 \\
1\end{array}$ & $\begin{array}{l}\text { Element } \\
\text { Element } \\
\text { Element or oxide }\end{array}$ \\
\hline
\end{tabular}


Table 1. Continued.

\begin{tabular}{|c|c|c|c|c|}
\hline Element & $\begin{array}{c}\text { Thickness Range } \\
\mathrm{g} / \mathrm{cm}^{2}\end{array}$ & $\begin{array}{l}\text { Method of } \\
\text { Preparation }\end{array}$ & Backing & $\begin{array}{l}\text { Form of } \\
\text { Deposit }\end{array}$ \\
\hline Europium & $\begin{array}{l}>10 \\
>2000 \\
\text { Variable }\end{array}$ & $\begin{array}{l}a \\
b \\
d\end{array}$ & $\begin{array}{l}2 \\
1 \\
1\end{array}$ & $\begin{array}{l}\text { El ement } \\
\text { Element } \\
\text { Element or oxide }\end{array}$ \\
\hline Fluorine & 25 to 500 & $a$ & 2 & Salt \\
\hline Gadol inium & $\begin{array}{l}>10 \\
>1000 \\
\text { Variable }\end{array}$ & $\begin{array}{l}a \\
b \\
d\end{array}$ & $\begin{array}{l}2 \\
1 \\
1\end{array}$ & $\begin{array}{l}\text { Element } \\
\text { Element } \\
\text { Element or oxide }\end{array}$ \\
\hline Gallium & $\begin{array}{l}10 \text { to } 200 \\
>10 \\
\text { Variable }\end{array}$ & $\begin{array}{l}a \\
a \\
d\end{array}$ & $\begin{array}{l}3 \\
2 \\
1\end{array}$ & $\begin{array}{l}\text { 0xide } \\
\text { Element } \\
\text { Dxide }\end{array}$ \\
\hline Germanium & $\begin{array}{l}>10 \\
>20 \\
\text { Variable }\end{array}$ & $\begin{array}{l}a \\
a \\
d\end{array}$ & $\begin{array}{l}2 \\
1 \\
1\end{array}$ & $\begin{array}{l}\text { Element } \\
\text { Eliement } \\
\text { Element or oxide }\end{array}$ \\
\hline Gold & $\begin{array}{l}>10 \\
\text { Variable } \\
\text { Variable } \\
\text { Variable }\end{array}$ & $\begin{array}{l}a \\
a \\
d \\
b\end{array}$ & $\begin{array}{l}2 \\
1 \\
1 \\
1\end{array}$ & $\begin{array}{l}\text { Element } \\
\text { Element } \\
\text { Element } \\
\text { Element }\end{array}$ \\
\hline Hafnium & $\begin{array}{l}\text { Variable } \\
\text { Variable }\end{array}$ & $\begin{array}{l}b \\
d\end{array}$ & $\begin{array}{l}1 \\
1\end{array}$ & $\begin{array}{l}\text { El ement } \\
\text { Element }\end{array}$ \\
\hline $\begin{array}{l}1_{H}, 2_{H} \text { or }{ }^{3} H \\
\text { absorbed on } \\
Z r, Y, T i, \text { or } E\end{array}$ & $\underline{-100}$ & e & 2 & $\begin{array}{l}\text { Hydride, deuteride, } \\
\text { or tritide }\end{array}$ \\
\hline Indium & $\begin{array}{l}>10 \\
>5000 \\
\text { Variable }\end{array}$ & $\begin{array}{l}a \\
b \\
d\end{array}$ & $\begin{array}{l}2 \\
1 \\
1\end{array}$ & $\begin{array}{l}\text { El ement } \\
\text { Element } \\
\text { Element }\end{array}$ \\
\hline Iridium & $\begin{array}{l}10 \text { to } 100 \\
\geq 10\end{array}$ & $\begin{array}{l}\mathrm{a} \\
\mathrm{e}\end{array}$ & $\begin{array}{l}2 \\
2\end{array}$ & $\begin{array}{l}\text { Element } \\
\text { Element }\end{array}$ \\
\hline Iron & $\begin{array}{l}>10 \\
>700 \\
\text { Variable }\end{array}$ & $\begin{array}{l}a \\
b \\
d\end{array}$ & $\begin{array}{l}2 \\
1 \\
1\end{array}$ & $\begin{array}{l}\text { El ement } \\
\text { El ement } \\
\text { Element }\end{array}$ \\
\hline Lanthanum & $\begin{array}{l}>10 \\
>1000 \\
\text { Variable }\end{array}$ & $\begin{array}{l}a \\
b \\
d\end{array}$ & $\begin{array}{l}2 \\
1 \\
1\end{array}$ & $\begin{array}{l}\text { Element } \\
\text { El ement } \\
\text { Element }\end{array}$ \\
\hline
\end{tabular}


Table 1. Continued.

\begin{tabular}{|c|c|c|c|c|}
\hline El ement & $\begin{array}{l}\text { Thickness Range } \\
\mathrm{g} / \mathrm{cm}^{2}\end{array}$ & $\begin{array}{l}\text { Method of } \\
\text { Preparation }\end{array}$ & Backing & $\begin{array}{l}\text { Form of } \\
\text { Deposit }\end{array}$ \\
\hline Lead & $\begin{array}{l}10 \text { to } 1000 \\
500 \text { to } 8000 \\
>6000 \\
\text { Tariable }\end{array}$ & $\begin{array}{l}a \\
a \\
b \\
d\end{array}$ & $\begin{array}{l}2 \\
1 \\
1 \\
1\end{array}$ & $\begin{array}{l}\text { El ement } \\
\text { El ement } \\
\text { El ement } \\
\text { Element }\end{array}$ \\
\hline Lithium & $\begin{array}{l}10 \text { to } 500 \\
10 \text { to } 500 \\
1000 \text { to any cast } \\
\text { size }\end{array}$ & $\begin{array}{c}a \\
a \\
b, d\end{array}$ & $\begin{array}{l}3 \\
2 \\
1\end{array}$ & $\begin{array}{l}\text { Hydride } \\
\text { El ement } \\
\text { El ement }\end{array}$ \\
\hline Lutetium & $\begin{array}{l}>10 \\
>1000 \\
\text { Variable }\end{array}$ & $\begin{array}{l}a \\
b \\
d\end{array}$ & $\begin{array}{l}2 \\
1 \\
1\end{array}$ & $\begin{array}{l}\text { Element } \\
\text { El ement } \\
\text { El ement or oxide }\end{array}$ \\
\hline Magnesium & $\begin{array}{l}>150 \\
>20,000 \\
\text { Variable }\end{array}$ & $\begin{array}{l}b \\
a \\
d\end{array}$ & $\begin{array}{l}1 \\
1 \\
1\end{array}$ & $\begin{array}{l}\text { El ement } \\
\text { El ement } \\
\text { El ement }\end{array}$ \\
\hline Manganese & $\frac{>10}{300}$ to 1600 & $\begin{array}{l}a \\
a\end{array}$ & $\begin{array}{l}2 \\
1\end{array}$ & $\begin{array}{l}\text { El ement } \\
\text { El ement }\end{array}$ \\
\hline Mercury & $\begin{array}{l}1000 \text { to } 4000 \\
\text { Variable }\end{array}$ & $\begin{array}{l}c \\
a\end{array}$ & $\begin{array}{c}\text { on } \mathrm{Au} \\
2\end{array}$ & $\begin{array}{l}\text { El ement } \\
\text { Salt }\end{array}$ \\
\hline Molybdenum & $\begin{array}{l}10 \text { to } 1000 \\
>500 \\
\text { Variable }\end{array}$ & $\begin{array}{l}a \\
b \\
d\end{array}$ & $\begin{array}{l}2 \\
1 \\
1\end{array}$ & $\begin{array}{l}\text { El ement } \\
\text { Element } \\
\text { Element or oxide }\end{array}$ \\
\hline Neodymi um & $\begin{array}{l}>10 \\
>1000 \\
\operatorname{Var} r^{2} \mathrm{able}\end{array}$ & $\begin{array}{l}a \\
b \\
d\end{array}$ & $\begin{array}{l}2 \\
1 \\
1\end{array}$ & $\begin{array}{l}\text { Element } \\
\text { El ement } \\
\text { Element or oxide }\end{array}$ \\
\hline Neptunium & $\begin{array}{l}>10 \\
\text { Variable }\end{array}$ & $\begin{array}{l}a \\
d\end{array}$ & 2,3 & $\begin{array}{l}\text { Oxide } \\
\text { Oxide }\end{array}$ \\
\hline Nickel & $\begin{array}{l}>10 \\
>500 \\
\text { Variable }\end{array}$ & $\begin{array}{l}a \\
b \\
d\end{array}$ & $\begin{array}{l}2 \\
1 \\
1\end{array}$ & $\begin{array}{l}\text { El ement } \\
\text { E T ement } \\
\text { El ement }\end{array}$ \\
\hline Niobium & $\begin{array}{l}>10 \\
\geq 700\end{array}$ & $\begin{array}{l}a \\
b\end{array}$ & $\begin{array}{l}2 \\
1\end{array}$ & $\begin{array}{l}\text { El ement } \\
\text { El ement }\end{array}$ \\
\hline Osmium & $\begin{array}{l}>10 \\
\text { Variable }\end{array}$ & $\begin{array}{l}a \\
d\end{array}$ & 2,3 & $\begin{array}{l}\text { El ement } \\
\text { Element }\end{array}$ \\
\hline
\end{tabular}


Table 1. Continued.

\begin{tabular}{|c|c|c|c|c|}
\hline Element & $\begin{array}{c}\text { Thi }{ }_{y / \mathrm{cm}^{2}} \\
\mathrm{y}^{2} \text { Range }\end{array}$ & $\begin{array}{l}\text { Method of } \\
\text { Preparation }\end{array}$ & Backing & $\begin{array}{l}\text { Form oi } \\
\text { Deposit }\end{array}$ \\
\hline Palladium & $\begin{array}{l}>10 \\
>1500 \\
\text { Variable }\end{array}$ & $\begin{array}{l}a \\
b \\
d\end{array}$ & $\begin{array}{l}2 \\
1 \\
1\end{array}$ & $\begin{array}{l}\text { El ement } \\
\text { Element } \\
\text { El ement }\end{array}$ \\
\hline Platinum & $\begin{array}{l}>10 \\
>1500 \\
\text { Variable }\end{array}$ & $\begin{array}{l}a \\
b \\
d\end{array}$ & $\begin{array}{l}2 \\
1 \\
1\end{array}$ & $\begin{array}{l}\text { El ement } \\
\text { El ement } \\
\text { Element }\end{array}$ \\
\hline Plutonium & $\begin{array}{l}>1 \\
25000 \\
\text { Variable }\end{array}$ & $\begin{array}{l}a \\
b \\
d\end{array}$ & $\begin{array}{l}2 \\
1 \\
1\end{array}$ & $\begin{array}{l}\text { Oxide } \\
\text { Element (1\% Al) } \\
\text { Oxide }\end{array}$ \\
\hline Potassium & $\begin{array}{l}7500 \text { to any cast } \\
\text { size }\end{array}$ & $b, d$ & 1 & Element \\
\hline Praseodymium & $\begin{array}{l}10 \text { to } 500 \\
1000 \text { to any cast } \\
\text { size } \\
\text { Variable }\end{array}$ & $\underset{d}{b, d}$ & $\begin{array}{l}2 \\
1 \\
1\end{array}$ & $\begin{array}{l}\text { Element } \\
\text { Element } \\
\text { Element or oxide }\end{array}$ \\
\hline Promethium & $\begin{array}{l}>10 \\
>10 \\
\text { Variable } \\
\text { Variable }\end{array}$ & $\begin{array}{l}a \\
a \\
b \\
d\end{array}$ & $\begin{array}{l}2 \\
2 \\
1 \\
1\end{array}$ & $\begin{array}{l}\text { Oxide } \\
\text { Element } \\
\text { Element } \\
\text { Element or oxide }\end{array}$ \\
\hline Rhenium & $\geq 10$ & a & 2,3 & Element \\
\hline Rubidium & $\geq 10$ & a & 2,3 & Element or salt \\
\hline Ruthenium & $\geq 1000$ & $f$ & 1 & Eiement in plastic \\
\hline Samarium & $\begin{array}{l}>10 \\
>1000 \\
\text { Variable }\end{array}$ & $\begin{array}{l}a \\
b \\
d\end{array}$ & $\begin{array}{l}2 \\
1 \\
1\end{array}$ & $\begin{array}{l}\text { El ement } \\
\text { Element } \\
\text { Element or oxide }\end{array}$ \\
\hline Scandium & $\begin{array}{l}>10 \\
>1000 \\
\text { Variable }\end{array}$ & $\begin{array}{l}a \\
b \\
d\end{array}$ & $\begin{array}{l}2 \\
1 \\
1\end{array}$ & $\begin{array}{l}\text { Element } \\
\text { Element } \\
\text { Oxide }\end{array}$ \\
\hline Selenium & $\begin{array}{l}10 \text { to } 5000 \\
300 \text { to } 5000 \\
\text { Variable }\end{array}$ & $\begin{array}{l}a \\
a \\
d\end{array}$ & $\begin{array}{l}2 \\
1 \\
1\end{array}$ & $\begin{array}{l}\text { El ement } \\
\text { El ement } \\
\text { El ement }\end{array}$ \\
\hline
\end{tabular}


Table 1. Continued.

\begin{tabular}{|c|c|c|c|c|}
\hline Eiement & $\begin{array}{c}\text { Thickness Range } \\
\mathrm{g} / \mathrm{cm}^{2}\end{array}$ & $\begin{array}{l}\text { Method of } \\
\text { Preparation }\end{array}$ & Backing & $\begin{array}{l}\text { Form of } \\
\text { Deposit }\end{array}$ \\
\hline Silicon & $\begin{array}{l}10 \text { to } 150 \\
10 \text { to } 1,000 \\
25 \text { to } 150 \\
50 \text { to } 400\end{array}$ & $\begin{array}{l}a \\
a \\
a \\
a\end{array}$ & $\begin{array}{l}2,3 \\
2 \\
1 \\
1\end{array}$ & $\begin{array}{l}\text { Oxide } \\
\text { Element } \\
\text { Oxide } \\
\text { Element }\end{array}$ \\
\hline Silver & $\begin{array}{l}10 \text { to } 1000 \\
100 \text { to } 5000 \\
>1000 \\
\text { Variable }\end{array}$ & $\begin{array}{l}a \\
a \\
b \\
d\end{array}$ & $\begin{array}{l}2 \\
1 \\
1 \\
1\end{array}$ & $\begin{array}{l}\text { El ement } \\
\text { El ement } \\
\text { El ement } \\
\text { El ement }\end{array}$ \\
\hline Sodium & $\begin{array}{l}7500 \text { to any cast } \\
\text { size }\end{array}$ & $b, d$ & 1 & Element \\
\hline Strontiun & $\geq 2000$ & b & 1 & Element \\
\hline Sulfur & $\begin{array}{l}10 \text { to } 100 \\
\geq 5000\end{array}$ & $\begin{array}{l}a \\
d\end{array}$ & $\begin{array}{l}2 \\
1\end{array}$ & $\begin{array}{l}\text { Element } \\
\text { Element }\end{array}$ \\
\hline Tantalum & $\begin{array}{l}10 \text { to } 1000 \\
\geq 1000\end{array}$ & $\begin{array}{l}a \\
b\end{array}$ & $\begin{array}{l}2 \\
1\end{array}$ & $\begin{array}{l}\text { El ement } \\
\text { El lement }\end{array}$ \\
\hline Technetium & 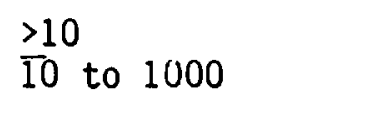 & $\begin{array}{l}c \\
a\end{array}$ & $\begin{array}{l}2 \\
2\end{array}$ & $\begin{array}{l}\text { El lement } \\
\text { El ement }\end{array}$ \\
\hline Tellurium & $\begin{array}{l}10 \text { to } 1000 \\
\text { Variable }\end{array}$ & $\begin{array}{l}a \\
d\end{array}$ & 2,3 & $\begin{array}{l}\text { El ement } \\
\text { El ement }\end{array}$ \\
\hline Terbium & $\begin{array}{l}>10 \\
>1000 \\
\text { Variable }\end{array}$ & $\begin{array}{l}a \\
b \\
d\end{array}$ & $\begin{array}{l}2 \\
1 \\
1\end{array}$ & $\begin{array}{l}\text { El ement } \\
\text { El ement } \\
\text { El ement or oxide }\end{array}$ \\
\hline Thallium & $\begin{array}{l}>10 \\
\geq 8000\end{array}$ & $\begin{array}{l}a \\
b\end{array}$ & $\begin{array}{l}2 \\
1\end{array}$ & $\begin{array}{l}\text { El ement } \\
\text { El ement }\end{array}$ \\
\hline Thorium & $\begin{array}{l}\geq 10 \\
\geq 4000\end{array}$ & $\begin{array}{l}a \\
b\end{array}$ & 2,3 & $\begin{array}{l}\text { El ement or oxide } \\
\text { El ement }\end{array}$ \\
\hline Thul ium & $\begin{array}{l}>10 \\
>1000 \\
\text { Variable }\end{array}$ & $\begin{array}{l}a \\
b \\
d\end{array}$ & $\begin{array}{l}2 \\
1 \\
1\end{array}$ & $\begin{array}{l}\text { El ement } \\
\text { El ement } \\
\text { Oxide }\end{array}$ \\
\hline Tin & $\begin{array}{l}10 \text { to } 1000 \\
100 \text { to } 4000 \\
>4000 \\
\text { Variable }\end{array}$ & $\begin{array}{l}a \\
a \\
b \\
d\end{array}$ & $\begin{array}{l}2 \\
1 \\
1 \\
1\end{array}$ & $\begin{array}{l}\text { El ement } \\
\text { El ement } \\
\text { El ement } \\
\text { El ement }\end{array}$ \\
\hline
\end{tabular}


Table 1. Continued.

\begin{tabular}{|c|c|c|c|c|}
\hline Element & $\begin{array}{c}\text { Thickness irange } \\
\mathrm{g} / \mathrm{cm}^{2}\end{array}$ & $\begin{array}{l}\text { Method of } \\
\text { Preparation }\end{array}$ & Backing & $\begin{array}{l}\text { Form of } \\
\text { Deposit }\end{array}$ \\
\hline Titanium & $\begin{array}{l}>10 \\
>500 \\
\text { Variable }\end{array}$ & $\begin{array}{l}a \\
b \\
d\end{array}$ & $\begin{array}{l}2 \\
1 \\
1\end{array}$ & $\begin{array}{l}\text { El ement } \\
\text { El ement } \\
\text { El ement }\end{array}$ \\
\hline Tungsten & $\begin{array}{l}>2,000 \\
\text { Variable }\end{array}$ & $\begin{array}{l}d \\
b\end{array}$ & $\begin{array}{l}1 \\
2\end{array}$ & $\begin{array}{l}\text { Element } \\
\text { Element (in } \mathrm{Cu} \\
\text { matrix) }\end{array}$ \\
\hline Uranium & $\begin{array}{l}>10 \\
>5000 \\
\text { Variable }\end{array}$ & $\begin{array}{l}a \\
b \\
d\end{array}$ & $\begin{array}{l}2 \\
1 \\
1\end{array}$ & $\begin{array}{l}\text { Oxide } \\
\text { Element } \\
\text { Element or oxide }\end{array}$ \\
\hline Vanadium & $\begin{array}{l}>10 \\
\frac{>1000}{\text { Variable }}\end{array}$ & $\begin{array}{l}a \\
b \\
d\end{array}$ & $\begin{array}{l}2 \\
1 \\
1\end{array}$ & $\begin{array}{l}\text { El ement } \\
\text { El ement } \\
\text { El ement }\end{array}$ \\
\hline Ytterbium & $\begin{array}{l}>10 \\
>2000 \\
\text { Variable }\end{array}$ & $\begin{array}{l}a \\
b \\
d\end{array}$ & $\begin{array}{l}2 \\
1 \\
1\end{array}$ & $\begin{array}{l}\text { El ement } \\
\text { El ement } \\
\text { Oxide }\end{array}$ \\
\hline Yttrium & $\begin{array}{l}>10 \\
>700 \\
\text { Variable }\end{array}$ & $\begin{array}{l}a \\
b \\
d\end{array}$ & $\begin{array}{l}2 \\
1 \\
1\end{array}$ & $\begin{array}{l}\text { El ement } \\
\text { El ement } \\
\text { El ement or oxide }\end{array}$ \\
\hline Zinc & $\begin{array}{l}>10 \\
>800 \\
\text { Variable }\end{array}$ & $\begin{array}{l}a \\
b \\
d\end{array}$ & $\begin{array}{l}2 \\
1 \\
1\end{array}$ & $\begin{array}{l}\text { El ement } \\
\text { El ement } \\
\text { Oxide }\end{array}$ \\
\hline Zirconium & $\begin{array}{l}10 \text { to } 5000 \\
>500 \\
\text { Variable }\end{array}$ & $\begin{array}{l}a \\
b \\
d\end{array}$ & $\begin{array}{l}2 \\
1 \\
1\end{array}$ & $\begin{array}{l}\text { El ement } \\
\text { Element } \\
\text { Element }\end{array}$ \\
\hline
\end{tabular}


Table 1. Continued.

NEUTRON DOSIMETERS

\section{Pure Materials}

\section{El ement}

$238 \mathrm{U}$

$235 \mathrm{U}$

237 Np

$239 \mathrm{pu}$

Sc

$\mathrm{Mn}$

${ }^{232}$ Th

$238 \mathrm{~J}$

$235 \mathrm{U}$

$239 p u$

$237 \mathrm{~Np}$

$233 \mathrm{v}$

Sc

Co

\section{Form}

$\mathrm{JO}_{2}$ ceramic oxide wire

$\mathrm{UO}_{2}$ ceramic oxide wire

$\mathrm{NpO}_{2}$ ceramic oxide wire

$\mathrm{PuO}_{2}$ ceramic oxide wire

$\mathrm{Sc}_{2} \mathrm{O}_{3}$ ceramic oxide wire

$\mathrm{Mn}_{3} \mathrm{O}_{4}$ ceramic oxide wire

$\mathrm{ThO}_{2}$ ceramic oxide wire

Dilute Materials

$0.5-1.0$ wt \% 238U-V alloy

$0.5-1.0$ wt \% 235U-V alloy

$0.5-1.0$ wt $\% \mathrm{Pu}$ as $\mathrm{PuO}_{2}-\mathrm{MgO}$ ceramic oxide wire

$0.5-1.0$ wt $\% \mathrm{~Np}$ as $\mathrm{NpO}_{2}-\mathrm{HgO}$ ceramic oxide wire

$0.5-1.0$ wt \% 233u-V alloy

0.1 wt $\% \mathrm{Sc}$ as $\mathrm{Sc}_{2} \mathrm{O}_{3}-\mathrm{MgO}$ ceramic oxide wire

0.1 wt $\%$ Co as CoO-MgO ceramic oxide wire 


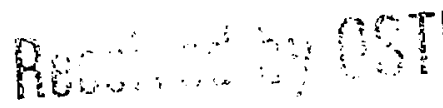

NOV 041986

\section{DISCLAIMER}

This report was prepared as an account of work sponsored by an agency of the United States Government. Neither the United States Government nor any agency thereof, nor any of their employees, makes any warranty, express or implied, or assumes any legal liability or responsibility for the accuracy, completeness, or usefulness of any information, apparatus, product, or process disclosed, or represents that its use would not infringe privately owned rights. Reference herein to any specific commercial product, process, or service by trade name, trademark. manufacturer, or otherwise does not necessarily constitute or imply its endorsement, recommendation, or favoring by the United States Government or any agency thereof. The views and opinions of authors expressed herein do not nccessarily statc or reflect those of the United States Government or any agency thereof. 